\title{
La narración del espectáculo político: pensar la relación entre sistema de medios y poder político
}

\author{
Natalia Aruguete \\ Universidad Nacional de Quilmes - CONICET
}

\section{Resumen}

Recibido: 15 de septiembre de 2013. Aceptado: 29 de octubre de 2013.

Dentro del campo de la comunicación, el debate acerca de la articulación entre el sistema de medios y el ámbito político se enriquece de diferentes corrientes teóricas. Diversos investigadores han estudiado la influencia mutua entre la agenda política y la agenda mediática, y los rastros que esta interacción deja en los textos noticiosos.

En la actualidad, definir a los medios como "actores políticos" parece una verdad de perogrullo. Sin embargo, el debate en torno al papel que estos juegan en el sistema político no está saldado. Menos aún, la problematización de su condición de actores políticos.

En este contexto, se propone una revisión bibliográfica que permita reflexionar sobre la participación activa de los medios de comunicación en el campo político. Concretamente, el objetivo de este artículo es, por un lado, sistematizar los aportes teóricos hechos a la interacción medios-política y, por otro, indagar la forma que toma esa tensa relación en la cobertura informativa de los asuntos de interés público, atendiendo especialmente a la narración de la noticia como espectáculo.

Palabras clave: medios de comunicación, actores políticos, sistema político, espectáculo político.

\section{The narration of political spectacle: considering the relationship between the media system and political power}

\section{Abstract}

Within the field of communication, different theoretical currents enrich the debate regarding the junctures between the media system and the political arena. Various investigators

\footnotetext{
1 La versión original de este artículo se expuso en el panel Perspectivas latinoamericanas sobre periodismo: escenarios y nuevos desafíos, celebrado en el marco del XV Congreso de la Red Argentina de Carreras de Comunicación Social y Periodismo (REDCOM), el 16 de agosto de 2013. Se agradecen los valiosos comentarios que el Dr. César Arrueta realizó a una primera versión de este artículo y se lo exime de toda responsabilidad respecto de los errores y omisiones que pudieran existir.
} 
have studied the mutual influence between political and media agendas, as well as the traces that this interaction leaves on news texts.

Nowadays, defining media as a "political actor" seems like a truism. Nevertheless, the debate about its role in the political system has not been closed. And neither has the problematization of its condition as a "political actor".

In this context, a bibliographical revision is proposed, which allows one to reflect on the active participation of communication media in the political field. Concretely, the objective of this paper is, on the one hand, to systematize theoretical contributions to the media-politics interaction, and on the other, to inquire into the shape adopted by this tense relationship in the press coverage of public interest topics, paying special attention to the narration of news as spectacle.

Keywords: : communication media, political actors, political system, political spectacle.

\section{A narração do espetáculo político: pensar a relação entre sistemas de meios e poder político.}

\section{Resumo}

O debate sobre a relação entre o sistema midiático e a arena política tem enriquecido por diferentes escolas teóricas do campo da comunicação. Vários pesquisadores têm estudado a influência mútua entre a agenda midiática e a agenda política, e os rastros desta interação nos textos noticiosos.

Atualmente, definir a mídia como "ator político" parece ser uma obviedade. Porém, o debate sobre o papel da mídia no sistema político não foi estabelecido. Muito menos ainda foi determinada a problematização da sua condição como ator político.

Neste contexto, nós propomos uma revisão da literatura para refletir sobre a participação ativa da mídia no campo político. Especificamente, o objetivo deste trabalho é sistematizar as contribuições teóricas feitas para a relação mídia-política. Ao mesmo tempo, procura-se pesquisar as maneiras em que essa tensa relação entre "mídia e política" se reflete no tratamento midiático das questões de interesse público até, mais precisamente, quando as histórias foram narradas espetacularmente.

Palavras-chave: mídia, atores políticos, sistema político, espetáculo político.

\section{Introducción. El rol de los medios en la política}

El proceso político define los requerimientos de información en cada momento histórico.

Bernard Cohen (1963, p. 6).

A los medios de comunicación se les suele atribuir un papel predominantemente pasivo. Son vistos como agencias neutrales que proveen un foro para el intercambio discursivo de otros actores, sin aportar su punto de vista. Nobleza obliga, circunscribir su función al de meros diseminadores de información no solo 
es una descripción deficiente sino, además, "una forma de distorsionar y subestimar el legítimo rol activo de los medios en el proceso de formación de la opinión pública" (Eilders, 2000, p. 183).

Los medios son un actor social y político necesario (Kircher, 2005); participantes diligentes con intereses y metas genuinos y con derecho a expresarlos en el discurso público en pos de encontrar resonancia (Eilders, 1997).

Selección, énfasis, omisión y encuadre ${ }^{2}$. Mediante estas estrategias discursivas, las instituciones periodísticas plasman en sus textos una "particular posición política” y un perfil ideológico que las distingue (Eilders, 2000, p. 181). Por ello, incorporar el análisis de los marcos en los que el discurso informativo se produce así como los que genera, supone tomar dimensión del rol fundamental que tales actores tienen en el juego político.

Los encuadres de las noticias son, sin más, la huella del ejercicio del poder: "registran la identidad de actores o intereses que compiten por dominar el texto" (Entman, 1993, p. 55). Claramente involucrados en tensiones políticas y socioeconómicas, los medios no podrían quedar exceptuados de esas huellas. Sobre todo, porque no operan con autonomía respecto del sistema político.

La doble relación que traban con el espacio público y el ámbito político se inserta en un proceso de acuerdos y disputas simbólicos alrededor de la generación de sentido(s) público(s), en el que cumplen un papel central. Nuevamente, los medios expresan sus intereses políticos y económicos y pugnan afanosamente por ellos. Pero aun cuando este sea un aspecto clave que se manifiesta en el escenario comunicacional, consideramos central entender que lo que prima es - fue y seguirá siendo- una lucha por el poder simbólico.

Los hallazgos obtenidos hasta el momento sobre la interacción medios-política no han llegado a un consenso. Algunos investigadores asumen que los medios juegan un rol considerable al causar la atención política hacia los asuntos (Trumbo, 1996; Soroka, Farnsworth, Lawlor, \& Young, 2012; Baumgartner, Bryan \& Leech, 1997). Otros advierten un efecto limitado de estos sobre la agenda política (Herman, 1993; Livingston, 1997; Mermin, 1997).

¿Qué sentido darle, cómo enmarcar la divergencia entre las tesis sostenidas aquí?

\footnotetext{
2 Desde la teoría del Encuadre (Framing theory), Stephen Reese, uno de sus principales exponentes, define "encuadre" como "principios organizadores socialmente compartidos y persistentes en el tiempo, que trabajan simbólicamente para estructurar el mundo social de modo significativo" (Reese, 2001, p. 11). En este artículo se utilizan los términos "encuadre" y "marco" indistintamente.
} 
Sin intenciones de eludir el debate, asumimos que el rol activo que las instituciones informativas tienen en el proceso político no les garantiza que su postura frente a ciertos temas tenga un real impacto en la agenda política.

¿De qué depende? De la centralidad dada a un tema y de que forme una opinión colectiva en el sistema mediático. Estos son aspectos que desarrollaremos en el próximo apartado.

\section{La consonancia entre los medios}

Los medios le importan a la política. Es inevitable. Están fuertemente involucrados en el proceso político e intervienen de distintas formas.

Ahora bien, ¿ todas las noticias tienen el mismo poder para influir en la agenda política? ¿Existen diferencias entre los medios a la hora de fijar los temas que preocuparán a los líderes políticos? Volvamos a los actores.

Para que los temas destacados por los medios tengan impacto en la agenda política es necesario un alto nivel de "congruencia" entre las coberturas informativas. En la medida en que las corporaciones periodísticas centren la atención en el mismo tema (focusing), lo encuadren en forma similar (consonance) y lo cubran con perseverancia (persistence), habrá más chances de generar efectos significativos sobre la arena política (Eilders, 1997).

La literatura llama centralidad (focusing) al grado de "correspondencia" entre los temas, en términos de su selección. La "consonancia", en cambio, alude a la dirección, al sentido de las opiniones existentes sobre un asunto.

El sistema de medios, afirma Christiane Eilders, actúa de manera "consonante" solo bajo ciertas circunstancias. Y advierte: en su propósito de diferenciarse de sus competidores, el foco puesto en determinados issues los identifica editorialmente; los distingue.

Coincidimos con la investigadora alemana, en parte.

La consonancia entre medios no va de suyo; debe ser analizada de manera "contextuada" (Arrueta, 2010). Hacemos hincapié en la necesidad de observar el contexto en el que los medios construyen su agenda ${ }^{3}$ y enmarcan su perspectiva sobre las cuestiones públicas, porque asumimos que "los mensajes noticiosos no pueden ser leídos, aprehendidos ni estudiados por fuera de las condiciones en que son producidos" (Aruguete, 2012, p. 90).

\footnotetext{
3 Kurt y Gladys Lang consideran más apropiado utilizar el término "construcción" que "establecimiento" de la agenda (Lang \& Lang, 1981).
} 
Porque además, la noticiabilidad ${ }^{4}$ que tiene un hecho tampoco le es inherente ni depende de sus rasgos intrínsecos. Gaye Tuchman, una de las primeras investigadoras en cuestionar la objetividad periodística como "ritual estratégico" (Tuchman, 1999), afirma que "la evaluación de la noticiabilidad es un fenómeno negociado, constituido por las actividades de una compleja burocracia diseñada para supervisar la red informativa" (Tuchman, 1983, p. 51).

La definición de Tuchman es elocuente para pensar la "consonancia temática" en un escenario en el que el papel de los productores mediáticos es puesto en cuestión, desacralizando la "inmaculada concepción" de los medios tradicionales (Becerra, 2011, p. 38). Frente a la visión funcionalista de que los medios son "organismos independientes y socialmente imparciales" se asiste a la ruptura de un consenso - que se había legitimado socialmente - según el cual "las empresas periodísticas disimulaban sus apuestas políticas bajo un discurso de neutralidad y objetividad" (Becerra, 2011, p. 37).

A las semejanzas encontradas en la cobertura de los distintos medios, que no solo publican los mismos tópicos sino que les dan un tratamiento similar, se las ha denominado "homogeneidad, consonancia o conformidad" (Bigman, 1948; Donohue \& Glasser, 1978; Riffe \& Shaw, 1982).

¿Por qué es tan fuerte esta tendencia de los medios a confirmar con otros el juicio periodístico sobre las noticias?

Porque tanto la adherencia a los canales rutinarios como la similitud de las historias permiten a los reporteros probarse a sí mismos que conocen la noticia real. “Consistencia es igual a precisión” (Reese \& Danielian, 1989, p. 34).

\section{La narración del espectáculo político}

La noticia no es una descripción de acontecimientos objetivos. Es una "ventana al mundo", afirma Tuchman. "La escena desarrollada depende de dónde se sitúa cada uno, lejos o cerca, estirando el cuello o mirando todo recto, con los ojos paralelos al muro o donde está la ventana" (Tuchman, 1983, p. 1).

La noticia como construcción se propone ante la sensibilidad pública y busca, a su vez, una reacción política. En este tirar de la cuerda, la espectacularización y el relato de las situaciones como crisis generalizadas suele contribuir a un mayor poder de agenda por parte de los medios. Este tipo de acontecimientos, sobre todo

\footnotetext{
4 Tomamos la definición que Wolf da de la "noticiabilidad" como los valores y criterios de selección, asociados a los procesos de rutinización y estandarización de las prácticas productivas de los medios (Wolf, 1991).
} 
cuando son definidos desde la lógica del espectáculo, tiene más probabilidades de ascender al juego de la política (Cobb \& Elder, 1971). "Cuando los eventos espectaculares están ausentes, los medios no pueden actuar uniformemente" (Bartels, 1996).

¿Qué es la narración del "espectáculo político"? Hablamos de espectáculo toda vez que la producción y difusión de las noticias están subordinadas a una lógica dramática.

El espectáculo constituido por las noticias continuamente construye y reconstruye los problemas sociales, las crisis, los enemigos y los líderes, creando de tal modo una sucesión de amenazas y seguridades. Esos problemas y personalidades construidos proveen el contenido del periodismo político y los datos para los estudios políticos, históricos y analíticos. También desempeñan un papel central en la obtención de apoyo y oposición a las diversas causas y políticas (Edelman, 1991, p. 8).

Hay tres rasgos de la lógica discursiva del espectáculo político, entre otros, que consideramos ilustrativos: la personalización, la dramatización y la descontextualización/simplificación. Nos centraremos en ellos.

En un intento voraz por atraer audiencias, las convenciones periodísticas han instalado una tendencia "irresistible" a focalizar su atención en los actores más que en sus acciones, en desmedro de los contextos en los que estas tienen lugar. La urgencia por meterse en la vida privada de celebridades y poner énfasis en actuaciones heroicas o escandalosas de personas comunes tiene un trasfondo ideológico: ignorar cuestiones estructurales (Edelman, 1991).

Desde este encuadre noticioso, la recurrencia a personajes e historias de vida -claramente más excitantes para el ojo público-genera una identificación en la audiencia que reacciona ante los "problemas" desde un enfoque moral e individual. Lo que queda oculto, sin dudas, es el panorama más estructural (institucional) que subyace al protagonismo de los muchos actores "atrapados en los ojos de la nueva cámara" (Bennett, 2012, p. 45). Claramente, concluye este autor, para los productores de la información es más "fácil" concentrarse en las vicisitudes de personas involucradas en combates políticos alrededor de las cuestiones que poner el foco en el poder y los procesos.

El poder parece ser entendido en un sentido limitado por los medios de comunicación [...]. Las historias hacen hincapié en la superficie de las apariencias, los sonidos furiosos y visiones exaltadas de batalla, las conocidas o coloridas personalidades involucradas [en cualquier situación que sea dramática]. Las causas subyacentes y el impacto real son poco observados y no serán recordados por mucho tiempo (Paletz \& Entman, 1981, p. 17). 
En el corto plazo, la personalización de la política — proliferación de héroes empaquetados en dramas políticos - ofrece una promesa de esperanza renovada, un mundo cargado de soluciones accesibles a los problemas políticos que marcan la vida en sociedad (Bennett \& Edelman, 1985).

En el largo plazo, menospreciar las posiciones social, económica y política en favor de los juicios humanos, las tragedias y los triunfos, genera cinismo y frustración. La sensación de "pérdida del poder alienta a la audiencia a abandonar el análisis político en favor del casting de los destinos del héroe del momento" (Aruguete, 2011).

La dramatización de la noticia suele ir acompañada de una escasa contextualización de los acontecimientos (Aruguete \& Amadeo, 2012). En efecto, el sesgo noticioso de la personalización aísla a los actores y sus acciones de un contexto social e institucional que les dé sentido.

Los aspectos de los eventos que se "denuncian" suelen ser expresados dramáticamente en "historias sencillas" (Bennett, 2012, p. 46), suprimiendo así la posibilidad de explicar los movimientos más estructurales del devenir social y político. En definitiva, el relato episódico ${ }^{5}$ despoja a los hechos de todo aquello que los haga comprensibles.

Un análisis contextualizado de los acontecimientos requeriría de explicaciones que hurguen en las causas de los asuntos. En cambio, el énfasis puesto en el drama y la acción, con la inevitable omisión de los cambios políticos, los vínculos temáticos entre los acontecimientos y los patrones históricos que los delimitan, provoca que la información pierda coherencia y continuidad.

Los dramas noticiosos enfatizan las crisis sobre las continuidades, el presente sobre el pasado y el futuro, poniendo a las personalidades en el centro de la escena. Minimizan la información política compleja, las actividades de las instituciones gubernamentales y las bases del poder detrás de los personajes principales (Bennett, 2012, p. 46).

Como en los melodramas, las noticias dramáticas arman escenarios polarizados, en los que es posible diferenciar a los agentes del bien de los del mal, a las víctimas de sus victimarios. Con el ojo puesto en el rating, se crean controversias

\footnotetext{
5 El encuadre episódico (episodic news frame) describe los asuntos públicos como instancias concretas o eventos definidos: un homeless, un desempleado, una víctima de discriminación racial, un intento de asesinato. En cambio, el encuadre temático (thematic news frame) se centra en temas políticos y eventos enmarcados en un contexto más abstracto: cambios en acciones políticas, la acumulación de un proceso de justicia criminal, la reducción de gastos gubernamentales. Este abordaje permite otorgar evidencias generales sobre los asuntos (Iyengar \& Kinder, 1987).
} 
-elemento constitutivo, por excelencia, del espectáculo político- alrededor de las decisiones políticas y de los rumbos económicos.

La determinación de responsabilidades, producto en parte del marco en el cual se da protagonismo a los actores, es clave: cuenta más la clara identificación de un actor político y su involucramiento en un tema - en tanto lo llama a actuar sobre este- que una atribución de responsabilidad ambigua (Pritchard, 1992).

En el mismo sentido, es más importante visualizar quién apoya y quién se opone a una determinada propuesta y el cálculo táctico de ese posicionamiento, que entender y precisar de qué se trataba la propuesta en cuestión. Y, sobre todo, qué consecuencias tendrá para el bienestar público (Edelman, 1991).

\section{Reflexiones finales}

La batalla por la opinión pública se juega en gran parte en los procesos de comunicación (Castells, 2008). Frente a esta tesis, ¿cuán simbiótica o controvertida se ha vuelto la interacción medios-política?

Algunos investigadores asumen que la política devino "política mediática", en tanto "el funcionamiento del sistema político se representa para los medios de comunicación" (Castells, 2008, p. 3). La "mediatización" es definida como una orientación de los actores y dinámicas políticas hacia los medios (Mazzoleni \& Schulz, 1999).

Esta hipótesis se asienta en la presunción de que existe una relación simbiótica según la cual, al tiempo que los políticos dependen del sistema de los medios de comunicación, las instituciones noticiosas transmiten y recrean las ideas dominantes del entorno cultural en el que operan, que van en el sentido construido desde y por los actores - políticos, económicos e institucionales- hegemónicos.

Otros, en cambio, advierten que en la interacción entre gobierno y medios, ambos campos son escenarios de lucha simbólica por el poder (Uranga, 2011) y que la cobertura mediática de los hechos está en íntima relación con el desarrollo político en el cual la prensa "es uno de los motores" (Lang \& Lang, 1981, p. 465).

En la Argentina no se hallaron evidencias empíricas sobre la relación entre medios y gobierno que excedan la mera indagación del uso y la subordinación de los líderes políticos a la lógica mediática para lograr una permanencia en el poder (Waisbord, 2013). Los pocos resultados encontrados remiten a la participación heterogénea de los actores involucrados en un acontecimiento en calidad de fuentes de información (Amadeo, 1999; Aruguete, 2011; Aruguete \& Zunino, 2013; Rey 
Lennon, 1998); un dato insuficiente, por cierto, si lo que se busca es conocer la correlación de fuerzas existente entre estos.

Más allá de la falta de constataciones empíricas, asumimos que la dramaticidad, la negatividad y la definición precisa de un asunto como problemático potencia la atención de los políticos hacia las coberturas. Los temas sensacionales, caracterizados por una baja complejidad en el relato de eventos dramáticos, otorgan más chances a los medios de liderar la agenda (Walgrave, Soroka \& Nuytemans, 2007).

La convicción de que los medios no operan con autonomía del sistema político nos permite inferir que la construcción de la agenda mediática surge de un proceso colectivo en el cual medios, gobiernos y ciudadanía se influyen recíprocamente en, al menos, algunos aspectos (Lang \& Lang, 1981).

Lejos estamos de suponer que las noticias tienen un efecto todopoderoso que performa percepciones públicas. Los medios no son el monolítico "gran hermano" que rige la atención pública (McCombs, 1996, p. 20). Sin embargo, presentar a la política como espectáculo sugiere un mensaje, no ya sobre lo que se cuenta sino sobre el interlocutor de ese relato.

Los hechos dignos de ser informados no existen independientemente de las personas que informan como de sus públicos. Las noticias no son descripciones asépticas de acontecimientos objetivos. Entonces, poco importan los temas puntuales de los que se hable; todas tienen un denominador común: ser catalizadoras de interpretaciones políticas sobre el mundo. Funcionan ante la sensibilidad del público y, desde allí, aceleran respaldo u oposición respecto de un determinado escenario.

Algunos de los mensajes que las noticias transmiten a sus públicos resultan elocuentes.

La generación de historias de "interés humano" (Edelman, 1991) honra la acción individual, pasando por alto cuestiones estructurales que puedan perturbar las ideologías. El espectáculo político, sostiene Edelman, no modifica creencias, las reafirma.

Se crean héroes o demonios efímeros. Frente a tal tratamiento episódico, la audiencia se ve compelida a proveer una historia y un futuro, dada su necesidad de explicarse los acontecimientos en términos de causas y consecuencias, de vincular una conducta observada con una persona a la que considera responsable de esa acción (Heider, 1930, 1958, 1978). Como consecuencia, en la memoria se fijan controversias y nombres distribuidos alrededor de esa polémica. 
Abordar el análisis político desde las causas y las consecuencias puede ser más importante, pero es sin dudas menos excitante. La "dificultad" de introducir antecedentes históricos en la información mediática invita a crear "un mundo de eventos caóticos y crisis que aparecen y desaparecen, porque las imágenes noticiosas ofrecen pocas explicaciones acerca de sus orígenes" (Bennett, 2012, p. 47).

En definitiva, la noticia dramatizada tiene implicancias políticas sobre la aptitud/actitud de las personas. Un desfile ilusorio de amenazas y seguridades tranquilizadoras, que no promueve una comprensión de los acontecimientos, sino que evoca un drama que objetiva esperanzas y temores. Más aún, le dice a la audiencia que su rol es menor, pasivo. A lo sumo, reactivo, pero no propositivo.

Genera un sentimiento de impotencia donde solo cabe la queja.

\section{Referencias}

Amadeo, B. (1999). La aplicación de la teoría del framing a la cobertura de la corrupción política en Argentina (1991-1996) [tesis doctoral]. Universidad de Navarra, Facultad de Comunicación, Pamplona, España.

Arrueta, C. (2010). ¿Qué realidad construyen los diarios? Buenos Aires: La Crujía.

Aruguete, N. (2011). The relationship between media and news sources: a content analysis of Argentine telephone service privatization in August to October 1990. Journal of Media and Communication Studies, 3 (9), p. 272-280.

Aruguete, N. (2012). Teorías de la opinión pública y de construcción de agendas. Buenos Aires: Universidad Nacional de Quilmes.

Aruguete, N. \& Amadeo, B. (2012). Encuadrando el delito: pánico moral en los periódicos Argentinos. América Latina Hoy, 62, p. 177-196.

Aruguete, N. \& Zunino, E. (2013). Diario Clarín y sus fuentes de información: un estudio de caso. Íconos, 46, p. 15-31.

Bartels, L. M. (1996). Politicians and the press: who leads, who follows? Paper prepared for APSA 1996, San Francisco, CA.

Baumgartner, F.; Bryan, D. \& Leech. B. (1997). Media attention and congressional agendas. En: Iyengar, S. \& Reeves, R. (eds.). Do the media govern?: politicians, voters, and reporters in America (p. 349-363). Thousand Oaks: Sage Publications.

Becerra, M. (2011). La inmaculada concepción de los medios latinoamericanos en crisis. Herramienta, 47, p. 29-40.

Bennett, W. (2012). News: the politics of illusion. Nueva York: Longman.

Bennett, W. \& Edelman, M. (1985). Toward a new political narrative. Journal of Communication, 35, p. $156-171$.

Bigman, S. (1948). Rivals y conformity: a study of two competing dailies. Journalism Quarterly, 25, p. 127-131.

Castells, M. (2008). Comunicación, poder y contrapoder en la sociedad red: I. Los medios y la política. Telos, 74, p. 13-24.

Cobb, R. \& Elder, T. (1971). The politics of agenda-building: an alternative perspective for modern democratic theory. Journal of Politics, 33, p. 892-915. 
Cohen, B. (1963). The press and foreign policy. Nueva York: Princenton University Press.

Donohue, T. \& Glasser, T. (1978). Homogeneity in coverage of Connecticut newspaper. Journalism Quarterly, 55, p. 592-596.

Edelman, M. (1991). La construcción del espectáculo político. Buenos Aires: Manantial.

Eilders, C. (1997). The impact of editorial content on the political agenda in Germany: theoretical assumptions and open questions regarding a neglected subject in mass communication research. Veröffentlichungsreihe der Abteilung Öffentlichkeit und Soziale Bewegungen des orschungsschwerpunkts Sozialer Wandel, Institutionen und Vermittlungsprozesse des Wissenschaftszentrums Berlin für Sozialforschung, p. 1-26. Disponible en internet: http://www. econstor.eu/bitstream/10419/49825/1/246100885.pdf

Eilders, C. (2000). Media as political actors?: issue focusing and selective emphasis in the German quality press. German Politics, 9 (3), p. 181-206.

Entman, R. (1993). Framing: toward a clarification of a fractured paradigm. Journal of Communication, 43 (3), p. 51-58.

Heider, F. (1930). Die Leistung des Wahnehmungssystems [The system of human perception]. Zeitschrift für Psychologie, 114, p. 371-394.

Heider, F. (1958). The psychology of interpersonal relations. Nueva York: Wiley.

Heider, F. (1978). Über balance und attribution [About balance and attribution]. En: Görlitz, D., Meyer, W. U. \& Weiner, B. (eds.). Bielefelder Symposium über Attribution (p. 19-28). Stuttgart: Klett.

Herman, E. (1993). The media's role in U.S. foreign policy. Journal of International Affairs, 47 (1), p. 23-46.

Iyengar, S. \& Kinder, D. (1987). News that matters: agenda setting and priming in a television age. Chicago: University of Chicago Press.

Kircher, M. (2005). La prensa escrita, actor social y político, espacio de producción cultural y fuente de información histórica. Revista de Historia. Neuquén: Universidad Nacional del Comahue, 10, p. 115-122.

Lang, G. \& Lang, K. (1981). Watergate: an exploration of the agenda-building process. Mass Communication Review Yearbook, 2, p. 447-468.

Livingston, S. (1997). "Beyond the "CNN-effect": the media-foreign policy dynamic". En: Norris, P. (ed.). Politics and the press: the news media and their influences (p. 291-314). Boulder, CO: Lynne Rienner.

Mazzoleni, G. \& Schulz, W. (1999). "Mediatization” of politics: a challenge for democracy? Political Communication, 16 (3), p. 247-61.

McCombs, M. (1996). La comunicación de masas en las campañas políticas: información, gratificación y persuasión. En: Moragas, M. de (ed.). Sociología de la comunicación de masas: estructura, funciones y efectos (p. 95-121). Barcelona: Gustavo Gili.

Mermin, J. (1997). Television news and the American intervention in Somalia: the myth of a mediadriven foreign policy. Political Science Quarterly, 112, p. 385-404.

Paletz, D. \& Entman, R. (1981). Media power politics. New York: Free Press.

Pritchard, D. (1992). “The news media and public policy agendas”. En: Kennamer, D. (ed.). Public opinion, the press and public policy (p. 103-112). Westport, CT: Praeger.

Reese, S. (2001). "Framing public life: a bridging model for media research". En: Reese, S., Gandy, O. \& Grant, A. (eds.). Framing public life: perspectives on media and our understanding of the social world (p. 7-31). Mahwah, NJ: Lawrence Erlbaum Associates.

Reese, S. \& Danielian, L. (1989). "Intermedia influence and the drug issue: converging on cocaine". En: Shoemaker, P. (ed.). Communication campaigns about drugs (p. 29-46). Hillsdale, NJ: Lawrence Erlbaum. 
Rey Lennon, F. (1998). Argentina: elecciones 1997: los diarios nacionales y la campaña electoral. Buenos Aires: Freedom Forum; Universidad Austral.

Riffe, D. \& Shaw, E. (1982). Conflict and consonance: converage of third world in two US papers. Journalism Quarterly, 59, p. 484-488.

Soroka, S.; Farnsworth, S.; Lawlor, A. \& Young, L. (2012). "Mass media and policymaking”. En: Araral, E., Fritzen, S., Howlett, M. Ramesh, M. \& Wu, X. (eds.). Routledge handbook of public policy. New York: Routledge.

Trumbo, C. (1996). Constructing climate change: claims and frames in US news coverage of an environmental issue. Public Understanding of Science, 5, p. 269-283.

Tuchman, G. (1983). La producción de la noticia: estudio sobre la construcción de la realidad. México, D.F.: Ediciones Gustavo Gili.

Tuchman, G. (1999). La objetividad como ritual estratégico: un análisis de las nociones de objetividad de los periodistas. CIC: Cuadernos de Información y Comunicación, 4, p. 199-218. Disponible en internet: http://revistas.ucm.es/index.php/CIYC/article/view/CIYC9899110199A/7407

Uranga, W. (2011). Entrecruzados: poder, política y comunicación. Herramienta, 15 (47), p. 41-50.

Waisbord, S. (2013). Cambios y continuidades: la agenda de investigación de la comunicación política en América Latina. Austral Comunicación, 2 (1), p. 105-131.

Walgrave, S.; Soroka, S. \& Nuytemans, M. (2007). The mass media's political agenda-setting power: a longitudinal analysis of media, parliament, and government in Belgium (1993 to 2000). Comparative Political Studies, 41 (6), p. 814-836.

Wolf, M. (1991). La investigación de la comunicación de masas. Buenos Aires: Paidós.

\section{Contacto}

\section{Natalia Aruguete}

nataliaaruguete@gmail.com 